\title{
Contribution à l'étude de la maladie des abcès chez le dromadaire (Camelus dromedarius) dans la région de Nefzaoua (sud-ouest de la Tunisie)
}

\author{
M.M. Seddik ${ }^{*}$ M.S. Ben Saîd ${ }^{2}$ M. Benzarti ${ }^{2}$ \\ T. Khorchani ${ }^{1}$ L. Messadi ${ }^{2}$ A. Amara ${ }^{2}$
}

Mots-clés

Camelus dromedarius Lymphadénite - Abcès Epidémiologie - Tunisie.

\section{Résumé}

Les résultats d'une enquête épidémiologique et clinique, et d'examen bactériologique relative à la maladie des abcès chez le dromadaire dans la région de Nefzaoua (sud-ouest de la Tunisie) sont présentés. L'étude épidémiologique a concerné 33 élevages en extensif ainsi que des dromadaires exploités dans le secteur touristique. Les études clinique et bactériologique ont porté sur 38 animaux issus de ces élevages. Cliniquement, la maladie n'a pas affecté l'état général de l'animal et a souvent touché les nœuds lymphatiques cervicaux inférieurs et préscapulaires en un nombre d'un ou deux abcès par animal atteint. La forme interne de la maladie n'a pas été observée sur les soixante-douze carcasses abattues dans I'abattoir de la région. La morbidité de la maladie dans la région a été de 9 p. 100. Cette maladie a souvent touché les jeunes dont l'âge était inférieur à un an. Les facteurs favorisants les plus incriminés dans l'apparition de la maladie ont été : le tatouage, le regroupement des animaux provenant de différents élevages et l'infestation par les tiques. Différents germes ont été isolés, notamment les staphylocoques (53 p. 100 des cas), Corynebacterium pseudotuberculosis (18 p. 100) et Actinomyces pyogenes (26 p. 100).

\section{INTRODUCTION}

Le dromadaire est l'animal domestique des régions arides et désertiques caractérisées par des parcours à faible productivité et par la rareté de l'eau. En Tunisie, cette espèce est devenue, pendant la dernière décennie, l'une des préoccupations majeures de l'élevage et constitue un axe de développement stratégique des régions du Sud. Plusieurs encouragements financiers et techniques, de même que certaines mesures d'accompagnement, ont été décidés et mis en œuvre afin de développer et promouvoir ce type d'élevage.

1. Laboratoire d'élevage et de la faune sauvage, Institut des régions arides, 4119 Médenine, Tunisie

2. Ecole nationale de médecine vétérinaire, 2020 Sidi-Thabet, Tunisie

* Auteur pour la correspondance

Tél. : +21675633005; fax : +21675633006

E-mail : seddik.mouldi@ira.rnrt.tn
L'aspect sanitaire occupe une place importante pour sauvegarder le cheptel et limiter l'extension de certaines maladies, notamment la gale et la trypanosomose. Une moindre importance est accordée aux autres affections, parmi lesquelles la maladie des abcès, appelée aussi la lymphadénite caséeuse, caractérisée par l'abcèdation des nœuds lymphatiques externes ou profonds. Cette maladie peut causer des pertes non négligeables chez le dromadaire comme chez d'autres espèces, mais n'a jamais été étudiée, à la connaissance des auteurs, en Tunisie. Elle a été décrite dans d'autres pays (réf. 1 à 14). Une enquête a été réalisée pour en connaître son importance.

\section{MATERIEL ET METHODES}

\section{Zone d'intervention}

L'enquête a été réalisée dans la région de Nefzaoua, au sud-ouest de la Tunisie. Elle a porté sur des troupeaux conduits en extensif ainsi que sur des dromadaires exploités dans le secteur touristique 
(sédentaires). Dans cette région, la conduite des troupeaux en mode extensif se caractérise par l'existence de deux périodes principales. Pendant la première, relativement longue, appelée hmil, qui s'étale du mois de mars jusqu'au mois d'octobre, les troupeaux sont libres dans le parcours (sans chamelier ni surveillance). Pendant la deuxième, qui s'étale du mois de novembre jusqu'à fin février, le troupeau se trouve sous la surveillance d'un ou deux chameliers qui gardent le troupeau puisque cette période coïncide avec la période du chamelage.

Les dromadaires exploités dans le secteur touristique ne sont pas conduits sur les pâturages comme les troupeaux de l'élevage. Ces animaux sont entretenus soit en un nombre réduit de deux à quatre têtes, soit individuellement à côté des maisons de leurs propriétaires. L'animal s'alimente en une seule prise au cours de la deuxième moitié de la journée. Cette ration peut être composée de fourrage à volonté, d'une complémentation comprenant $4 \mathrm{~kg}$ d'orge (parfois remplacée par du son de blé) et $2 \mathrm{~kg}$ de dattes ou de noyaux de dattes. L'abreuvement est de fréquence variable selon la température : une fois tous les trois quatre jours pendant la saison froide, quotidiennement en été.

\section{Troupeaux et animaux}

L'enquête effectuée a porté sur 33 élevages en extensif, situés dans les différentes régions de cette zone, comptant au total 3259 têtes. Les animaux conduits en mode sédentaire appartenaient aux syndicats touristiques de la région situés à Douz et Zaâfrane et étaient répartis en trois groupes, chacun composé de 200 à 350 têtes, soit un total de 900 têtes.

\section{Matériel de prélèvement}

Le matériel suivant a été utilisé : des tubes de sang Edta ; des tubes d'écouvillonnage stériles, des seringues et des aiguilles métalliques, des petits matériels de chirurgie (ciseaux, pinces, bistouri, sonde...), des compresses et du coton, et des flacons d'antiseptiques (Poliodine). Les prélèvements ont été maintenus au froid.

\section{Sur le terrain \\ Examen clinique}

Pour chaque élevage a été réalisé un examen sur l'ensemble des animaux dans un premier temps. Les animaux présentant des hypertrophies ganglionnaires ont fait l'objet d'un examen clinique approfondi sur les grandes fonctions.

\section{Prélèvements}

Les prélèvements du pus ont été effectués sur des abcès mûrs, non ouverts. La ponction a été faite à l'aide d'une seringue, après avoir rasé et désinfecté la surface externe de l'abcès. Le pus prélevé a été vidé dans le tube d'écouvillonnage. Après prélèvement, un traitement chirurgical par incision en forme de « $\mathrm{T}$ » de l'abcès, suivie d'une vidange, nettoyage et lavage par les antiseptiques a été fait. Le prélèvement du sang a été réalisé à partir de la veine jugulaire, dans des tubes Edta.

\section{Enquête épidémiologique}

Un questionnaire a été réalisé avec le chamelier portant sur le troupeau et le ou les animaux atteints, en vue d'analyser la ou les causes favorisantes de la contamination.

\section{A l'abattoir}

Après examen des nœuds lymphatiques externes, la forme interne de la maladie a été recherchée sur les viscères. D'autre part, des nœuds lymphatiques externes atteints ont été prélevés pour l'examen histologique. Une préfixation a été faite par le liquide de Boin pour la conservation des prélèvements.

\section{Au laboratoire \\ Examen bactériologique du pus}

Après un examen macroscopique du pus (consistance, couleur et odeur), les prélèvements ont été ensemencés sur une gélose au sang de mouton et incubés pendant $48 \mathrm{~h}$ à $37^{\circ} \mathrm{C}$ en anaérobiose. Les boîtes ont été examinées visuellement pour mettre en évidence la taille, l'opacité, la pigmentation, la morphologie des colonies et la présence ou l'absence d'hémolyse. Chaque type de colonie a fait l'objet d'une coloration de Gram. L'identification des germes a été faite par les tests suivants : la catalase, le milieu de Chapman, la coagulase sur plasma de lapin et la détection de la désoxyribonucléase sur gélose à l'Adn, pour les coques Gram positif. La catalase, le milieu Kligler Hajna, le milieu urée indol et le mannitol mobilité ont été utilisés pour les bacilles Gram positif et l'oxydase pour les bacilles Gram négatif.

\section{Examens histologique et hématologique}

Les nœuds lymphatiques prélevés ont été fixés par le formol à 10 p. 100 pendant $24 \mathrm{~h}$, puis inclus dans la paraffine et coupé en fines lamelles de trois microns d'épaisseur à l'aide d'un microtome. Ces coupes ont été montées ensuite entre lame et lamelle après coloration à l'hémalun-éosine. Pour chaque animal atteint, un hémogramme a été réalisé à partir des prélèvements sanguins.

\section{RESULTATS ET DISCUSSION Observation clinique}

La maladie a évolué selon un mode chronique, affectant surtout les jeunes de moins de cinq ans avec une morbidité maximale chez les chamelons d'un an. Ces animaux ont présenté principalement une atteinte des nœuds lymphatiques cervicaux inférieurs ainsi que des nœuds préscapulaires. Un ou deux abcès ont été observés par animal atteint. La lésion avait une taille variable selon les localisations, en général elle prenait la taille d'une orange $(10 \mathrm{~cm}$ de diamètre). Ces observations ont été comparables à celles décrites par Moustafa (9) qui concernaient quatre dromadaires adultes dans un troupeau de 150 têtes en Libye. La lésion commence par l'augmentation de la taille avec collection du pus puis on observe une chute des poils au sommet de la lésion, elle devient molle. En absence de traitement, l'abcès s'ouvre et par une fistule le pus s'élimine en partie sur l'entourage de la lésion ; l'ensemble faisant une couche difficilement détachable conduisant parfois à la nécrose cutanée. La maladie n'a pas affecté les grandes fonctions ni l'état général de l'animal atteint.

Sur 72 carcasses de dromadaires abattus à l'abattoir de la région, aucune lésion interne de la maladie n'a été observée. Seuls certains chameliers ont rapporté la forme interne qui affecte l'état général et cause parfois la mort. A l'abattage des animaux, malades, les chameliers ont constaté la présence d'abcès viscéraux de tailles variables au poumon et au cœur, mais cette forme a été très rare. Ceci est en accord avec les travaux d'Anderson (2) qui a signalé cette forme interne rare.

\section{Résultat épidémiologique Epidémiologie descriptive}

Dans la région de Nefzaoua, la maladie est appelée Khraja par les chameliers. Les élevages visités appartenaient à plusieurs éleveurs de la région qui se concentraient pendant la période de l'enquête sur les parcours à plantes halophiles, plantes préférées par le dromadaire pendant la période hivernale. 
La majorité des troupeaux visités ont été affectés par la maladie. Seul deux troupeaux, où les jeunes étaient absents, ont été indemnes. Ceci laisse supposer une prédisposition des jeunes à la maladie, puisque 23 p. 100 d'entre eux ont été atteints (tableau I). Les deux sexes on été touchés sans différence significative.

Selon les chameliers la morbidité maximale a été observée pendant l'automne, entre septembre et décembre, période où les animaux sont mieux surveillés. Aucun traitement traditionnel n'a été utilisé contre la maladie. Les abcès se sont fistulés spontanément, laissant couler le pus pendant des périodes variables et évoluant vers la cicatrisation ou se compliquant parfois par de la nécrose cutanée.

\section{Epidémiologie analytique}

La maladie a été présente dans 94 p. 100 des élevages visités en extensif. Parmi les traumatismes inoculateurs recherchés, l'infestation par les tiques a semblé être un facteur très important dans la région pour la transmission de la maladie (tableau II). Les injections médicamenteuses et les parcours épineux semblent avoir eu une moindre importance du fait que la peau du dromadaire est épaisse, notamment chez les adultes. La prédisposition des jeunes à la maladie pourrait être liée, en effet, à leur peau plus mince.

Pour les facteurs de risque, le regroupement des animaux très fréquent entre les différents troupeaux de la région, notamment lors d'abreuvement ou lors de travail pour les dromadaires exploités dans le secteur touristique, a semblé prépondérant. Pour les autres facteurs recherchés, l'ouverture des abcès sans traitement a constitué un facteur important dans la dissémination de la maladie au sein du troupeau.

Pour la conduite d'élevage, du fait qu'elle est saisonnière dans la région de Nefzaoua, les troupeaux subissent un seul traitement collectif contre les parasites externes par l'utilisation des produits acaricides, d'où le manque d'hygiène pendant la fin de printemps. L'été semble favoriser la dissémination de la maladie. Cette période coïncide avec la pleine activité des insectes et les acariens.

\section{Résultats bactériologiques et histopathologiques}

Sur un total de 38 prélèvements, 24 en extensif et 14 sédentaires, Corynebacterium pseudotuberculosis a été isolé dans sept cas alors que d'autres germes pyogènes ont été isolés, notamment les staphylocoques (53 p. 100 des cas) et Actinomyces pyogenes (26 p. 100) (tableau III). Corynebacterium pseudotuberculosis n'a été isolé qu'à partir des prélèvements effectués sur des animaux sédentaires donc sur les sujets adultes dont l'âge a varié de 3 à 14 ans. Staphylococcus spp. et Actinomyces pyogenes ont été isolés dans les deux types élevages. La maladie des abcès chez le dromadaire est donc apparue causée par plusieurs agents infectieux, ce qui concorde avec d'autres travaux (tableau IV).

L'hématologie des animaux atteints a été caractérisée par une hyperleucocytose dont la valeur a été de $22000 / \mathrm{mm}^{3}$. D'autre part, l'anémie a été remarquée chez huit sujets malades dont les taux d'hémoglobines ont varié entre 7 et $9,2 \mathrm{~g} / \mathrm{dl}$, vraisemblablement due à d'autres affections existant chez les animaux examinés. L'histologie de la lésion a montré la présence d'un centre nécrotique entouré d'une membrane pyogène formée par un infiltrat polymorphe (granulocytaire et lympho-plasmocytaire), l'ensemble étant entouré par une coque fibreuse.

\section{Tableau I}

Répartition des animaux atteints en fonction de l'âge et selon le mode d'élevage

Jeunes ( $\leq 1$ an) Adultes ( $>1$ an)

\section{Elevage extensif}

$\begin{array}{lcc}\text { Nb. total d'animaux } & 705 & 2554 \\ \text { Nb. d'animaux atteints } & 164 & 111 \\ \% & 23,26 & 4,34\end{array}$

Elevage sédentaire

$\begin{array}{llc}\text { Nb. total d'animaux } & 0 & 900 \\ \text { Nb. d'animaux. atteints } & 0 & 15 \\ \% & 0 & 1,66\end{array}$

Tableau II

Facteurs de risques observés chez les troupeaux présentant des abcès

\begin{tabular}{|c|c|c|c|}
\hline & Elevage & Oui (\%) & Non $(\%)$ \\
\hline \multicolumn{4}{|l|}{ Facteurs de risques } \\
\hline \multirow[t]{2}{*}{ Infestation par les tiques } & Extensif & $31(100)$ & $0(0)$ \\
\hline & Sédentaire & $12(100)$ & $0(0)$ \\
\hline \multirow[t]{2}{*}{ Pratique du tatouage } & Extensif & $24(77)$ & $7(23)$ \\
\hline & Sédentaire & $10(83)$ & $2(17)$ \\
\hline Application des & Extensif & $12(38)$ & $18(62)$ \\
\hline boucles d'oreilles & Sédentaire & $0(0)$ & $12(100)$ \\
\hline \multicolumn{4}{|l|}{ Facteurs favorisants } \\
\hline \multirow[t]{2}{*}{ Ouverture des abcès } & Extensif & $26(83)$ & $5(17)$ \\
\hline & Sédentaire & $7(58)$ & $5(42)$ \\
\hline \multirow{2}{*}{$\begin{array}{l}\text { Regroupement } \\
\text { des troupeaux }\end{array}$} & Extensif & $31(100)$ & $0(0)$ \\
\hline & Sédentaire & $12(100)$ & $0(0)$ \\
\hline
\end{tabular}

Tableau III

Germes isolés

\begin{tabular}{|c|c|c|c|c|c|c|}
\hline Elevage & $\begin{array}{l}\text { Corynebacterium } \\
\text { pseudotuberculosis }\end{array}$ & $\begin{array}{c}\text { Actinomyces } \\
\text { pyogenes }\end{array}$ & $\begin{array}{l}\text { Staphylococcus } \\
\text { epidermedis }\end{array}$ & $\begin{array}{c}\text { Staphylococcus } \\
\text { intermedius }\end{array}$ & $\begin{array}{l}\text { Escherichia } \\
\text { coli }\end{array}$ & Total \\
\hline Extensif & 0 & 8 & 11 & 4 & 1 & 24 \\
\hline Sédentaire & 7 & 2 & 4 & 1 & 0 & 14 \\
\hline Total & 7 & 10 & 15 & 5 & 1 & 38 \\
\hline
\end{tabular}




\section{Tableau IV}

Analyses bibliographiques des différents germes isolés

\begin{tabular}{|c|c|c|}
\hline Origine & Germe & Référence \\
\hline Dromadaires atteints en Ethiopie & $\begin{array}{l}\text { Deux types de germes : } \\
\text { les corynébactéries et les streptocoques }\end{array}$ & Richard, 1975, université de Créteil \\
\hline $\begin{array}{l}\text { Analyses sur } 4 \text { dromadaires atteints } \\
\text { en Libye }\end{array}$ & Actinomyces pyogènes & Moustafa, 1994, Revue Elev. Méd. vét. Pays trop. \\
\hline Dromadaires atteints en Ethiopie & $\begin{array}{l}\text { Corynebacterium pseudotuberculosis } \\
\text { et Streptococcus groupe B }\end{array}$ & $\begin{array}{l}\text { Domenech et coll., 1977, Revue Elev. Méd. vét. } \\
\text { Pays trop. }\end{array}$ \\
\hline $\begin{array}{l}\text { Analyses sur } 11 \text { cas de lymphadénite } \\
\text { en Egypte }\end{array}$ & Corynebacterium pseudotuberculosis & Afzal et coll., 1996, Trop. Anim. Health Prod. \\
\hline Analyse sur un dromadaire & Corynebacterium ulcerans & Tejedor et coll., 2000 (site Web) \\
\hline $\begin{array}{l}\text { Camélidés en Amérique de Nord } \\
\text { Abcès contagieux } \\
\text { Abcès non contagieux }\end{array}$ & $\begin{array}{l}\text { Corynebacterium pseudotuberculosis } \\
\text { Actinomyces pyogenes } \\
\text { Streptococcus spp. } \\
\text { Staphylococcus spp. } \\
\text { Fusobacterium spp. }\end{array}$ & Anderson, 2000 (site Web) \\
\hline $\begin{array}{l}\text { Analyses à partir des abcès cutanés } \\
\text { chez des dromadaires atteints en Egypte }\end{array}$ & $\begin{array}{l}\text { Staphylococcus } \\
\text { Actinomyces pyogenes } \\
\text { Clostridium perfringens } \\
\text { Escherichia coli } \\
\text { Pseudomonas aeroginosa } \\
\text { Corynebacterium pseudotuberculosis } \\
\text { Fusobacterium necrophorum } \\
\text { Klebsiella }\end{array}$ & Ismail et coll., 1990, Vet. Med. J. Giza \\
\hline
\end{tabular}

\section{CONCLUSION}

Du fait que cette maladie touche de préférence les sujets jeunes et qu'elle peut conduire à une atteinte de l'état général, la lutte s'avère nécessaire. La vulgarisation auprès des chameliers, leur formation et leur équipement en produits antiseptiques nécessaires pourraient être les solutions pour éviter les complications possibles des fistules, surtout lorsqu'on sait qu'il est très difficile d'accéder et de localiser un troupeau sur le terrain à cause de sa mobilité.

Le mode sédentaire d'élevage semble favoriser la survie de $C$. pseudotuberculosis qui entraînerait la forme contagieuse d'après Anderson (2) et qui pourrait persister longtemps en milieu extérieur, alors qu'en élevage extensif, il est très rare de trouver un animal adulte atteint par la maladie. Cependant, l'éradication de la maladie paraît difficile, compte tenu de la grande résistance des germes dans le milieu extérieur et de la multiplicité des espèces réceptives, notamment les ovins et les caprins. Par contre, il est possible de limiter l'infection par la bonne conduite sanitaire des troupeaux.

\section{BIBLIOGRAPHIE}

1. AFZAL M., SAKIR M., MAIID H.M., 1996. Corynebacterium pseudotuberculosis infection and lymphadenitis (taloa or mala) in the camel. Trop. Anim. Health Prod., 28: 158-162.

2. ANDERSON D.E., 2000. Contagious abscesses in camelids. W.W.W.vet.Ohio-state.edu /docs/clin.sci/camelid/abscess.htm

3. CURASSON G., 1947. Le chameau et ses maladies. Paris, France, Vigot frères, $462 \mathrm{p}$.

4. DEWITT K., 1999. Caseous lymphadenitis is a very contagious disease that causes internal and external abscesses. Eradication is difficult and vaccination has shown to be helpful in management of the disease in infected herds. W.W.W.ogdomain.com/web/silhouettepygmygaets/cl

5. DOMENECH J., GUIDOT G., RICHARD D., 1977. Les maladies pyogènes du dromadaire en Ethiopie : symptomatologie - étiologie. Revue Elev. Méd. vét. Pays trop., 30 : 251-258.

6. FAYE B., 1997. Guide de l'élevage de dromadaire. Libourne, France, Sanofi, $126 \mathrm{p}$.

7. HIGGINS A.J., 1986. The camel in health and diseases. London, UK, Bailliere Tindall, $168 \mathrm{p}$.

8. ISMAIL M., EZZAT M., EL-JAKEE J., EL-SAYED Z.E., ABDERAHMEN M., 1990. Microorganisms associated with closed abscesses of camels in Egypt. Vet. Med. J. Giza, 38: 53-62.

9. MOUSTAFA A.M., 1994. First observation of camel (Camelus dromedarius) lymphadenitis in Libya: a case rapport. Revue Elev. Méd. vét. Pays trop., 47: 313-314.

10. RICHARD D., 1975. Etude de la pathologie du dromadaire dans la sous-province de Borana (Ethiopie). Thèse Doct. vét., université de Créteil, France, 181 p. ( $\left.\mathrm{N}^{\circ} 75\right)$

11. SCHWARTZ H.J., DIOLI M., EDS, 1992. The one-humped camel in Eastern Africa. A pictorial guide to diseases, health care and management. Weikersheim, Germany, Verlag Josef Margraf, 282 p.

12. TEJEDOR M.T., MARTIN J.L., LUPIOLA P., GUTIERREZ C., 2000. Caseous lymphadenitis caused by Corynebacterium ulcerans in the dromedary camel. W.W.W.cvma.acmv.org/vet journals/cvj/contents 00/feb-toc00.htm

13. WALKER J., JACKSON H.J., BRANDON M.R., MEEUSEN E.N.T., 1991. Lymphocyte subpopulations in pyogranulomas of caseous lymphadenitis. Clin. exp. Immunol., 86: 13-18.

14. WERNERY U., KAADEN O.R., 1997. Contagious diseases in the dromedary camel. Berlin, Germany, Blackwell Wissenschafts, $110 \mathrm{p}$.

Reçu le 03.04.2003, accepté le 29.03.2004 


\section{Summary}

Seddik M.M., Ben Said M.S., Benzarti M., Khorchani T., Messadi L., Amara A. Contribution to the Study of Abscess Disease in the Dromedary (Camelus dromedarius) in Nefzaoua Region (southwest of Tunisia)

Results of an epidemiological, clinical and bacteriological survey about the abscesses disease in Nefzaoua region (southwest of Tunisia) are presented. It concerned 33 herds in extensive conditions and also animals exploited in the touristic sector. Clinical and bacteriological examinations were carried out on 38 animals from these herds. The disease was generally characterized by one or two abscesses in the external lymph nodes, usually in those at the base of the neck, and the prescapular nodes. The internal form of the disease was not observed in 72 camels slaughtered at the region slaughterhouse. Nine per cent of the animals had abscesses. Generally, the disease was observed in the young less than one year old. The main factors that caused onset of the disease were tattooing, mixing animals from different herds and tick infection. Different bacteria were isolated, in particular staphylococci (53\% of cases), Corynebacterium pseudotuberculosis (18\%) and Actinomyces pyogenes (26\%).

Key words: Camelus dromedarius - Lymphadenitis - Abscess Epidemiology - Tunisia.

\section{Resumen}

Seddik M.M., Ben Said M.S., Benzarti M., Khorchani T., Messadi L., Amara A. Contribución al estudio de la enfermedad de los abscesos en el dromedario (Camelus dromedarius) en la región de Nefzaoua (sudoeste de Túnez)

Se presentan los resultados de una encuesta epidemiológica y clínica y del examen bacteriológico relativo a la enfermedad de los abscesos en el dromedario en la región de Nefzaoua (sudoeste de Túnez). El estudio epidemiológico concernió 33 establecimientos extensivos, así como dromedarios explotados en el sector turístico. Los estudios clínicos y bacteriológicos se llevaron a cabo en 38 animales de estos establecimientos. Clínicamente, la enfermedad no afectó el estado general del animal y a menudo afectó los nódulos linfáticos cervicales inferiores y pre-escapulares, con uno o dos abscesos por animal afectado. La forma interna de la enfermedad no fue observada en las 72 carcasas sacrificadas en el matadero de la región. La morbilidad de la enfermedad en la región fue de $9 \%$. Esta enfermedad afecta a menudo los jóvenes menores de un año de edad. Los factores favorecedores más incriminados en la aparición de la enfermedad fueron: el tatuaje, el agrupamiento de los animales provenientes de diferentes crías y la infestación por garrapatas. Se aislaron diferentes gérmenes, principalmente estafilococos (53\%), Corynebacterium pseudotuberculosis (18\%) y Actinomyces pyogenes (26\%).

Palabras clave: Camelus dromedarius - Linfoadenitis Absceso - Epidemiología - Túnez. 